\title{
Metastatic melanoma in the Mid-West of Ireland: a retrospective review
}

\author{
Emma Porter ${ }^{1}$ - Irene Timoney ${ }^{1} \cdot$ Berbie Byrne $^{1} \cdot$ Vivien Marasigan $^{1} \cdot$ Caitriona Hackett $^{1} \cdot$ Bart Ramsay $^{1}$. \\ Grzegorz Korpanty ${ }^{2} \cdot K_{\text {Kashif Ahmad }}{ }^{1}$
}

Received: 20 October 2021 / Accepted: 25 December 2021 / Published online: 8 January 2022

(c) The Author(s), under exclusive licence to Royal Academy of Medicine in Ireland 2022

\begin{abstract}
Background Melanoma is the fifth most common invasive cancer in Ireland, and incidence is increasing. Metastatic melanoma has been associated with poor overall survival historically. New systemic anti-cancer treatment (SACT) options for advanced melanoma have emerged in the last decade, and outcomes are improving.

Aims The aim of our study was to assess the incidence and clinicopathological features of metastatic melanoma in our centre, and subsequent treatment with SACT.

Methods We analysed retrospectively patients with metastatic melanoma in the Mid-West of Ireland, over a 6-year period (2014-2019).

Results In 6 years, a total of 620 patients were diagnosed with melanoma, 28 (5\%) had metastatic or unresectable disease at diagnosis. Mean age at primary diagnosis was 64.5 years (range 24-90 years) and 20 (71\%) were male. Median Breslow depth was $4.3 \mathrm{~mm}$ (mean $5.5 \mathrm{~mm}, \mathrm{SD} \pm 4.4 \mathrm{~mm}$ ). Thirteen patients (46\%) had metastases at initial presentation. Fifteen (53\%) received systemic treatment in the regional cancer centre. Of 13 who did not have systemic treatment, 8 had radiological and clinical surveillance, 3 declined further treatment or surveillance and 2 were lost to follow-up. Eleven patients died from the disease with median overall survival of 1.5 years $(\mathrm{SD} \pm 1.3$ years).

Conclusion Patients with metastatic melanoma commonly had metastases at the time of first presentation. Just over half of patients with metastatic melanoma received SACT. Early detection of melanoma is key. Further research on factors involved in late presentation, and those precluding systemic treatment, may contribute to improved outcomes in advanced melanoma.
\end{abstract}

Keywords Dermatology $\cdot$ Medical oncology $\cdot$ Melanoma $\cdot$ Skin cancer

\section{Background}

Malignant melanoma is the fifth most common invasive cancer in Ireland, and incidence is increasing with approximately 20 cases per 100,000 per year [1]. Ireland has one of the highest recorded mortality rates for melanoma in Europe. Data available from the European Cancer Information System has demonstrated that Ireland had the third highest agestandardised mortality rate (ASR) per 100,000 for melanoma in the year 2014, and the highest ASR in 2013 [2]. While

Emma Porter

emma.mi.porter@gmail.com

1 Department of Dermatology, University Hospital Limerick, Limerick, Ireland

2 Medical Oncology Department, University Hospital Limerick, Limerick, Ireland the vast majority of patients are diagnosed and treated at an early stage, one fifth of patients are at stage III or IV at the time of diagnosis. This proportion of late-stage tumours remained stable over time according to National Cancer Registry Ireland data for the years 1994-2013 [1].

Metastatic disease has historically been associated with poor survival [3]. Ten years ago, median overall survival of those with advanced-stage melanoma was 9 months [4]. With the advent of new systemic anti-cancer therapy (SACT) for the treatment of metastatic melanoma in the last decade, outcomes are vastly improving [5]. Targeted treatment for $B R A F$-mutated melanoma has been a particular area of advancement, with approaches to treatment shifting towards combined BRAF and MEK inhibition. Immunotherapy options have also expanded, with availability of immune checkpoint inhibitors including anti-cytotoxic T-lymphocyte-associated antigen-4 (CTLA4), such as ipilimumab, and programmed cell-death protein 
1 (PD-1) inhibitors such as pembrolizumab and nivolumab. Recent studies suggest that even functional cure may be possible, considered when patients have responded to treatment and remained without disease progression after 2 years treatmentfree [5].

The National Cancer Control Programme published a detailed overview of recommendations in 2017 on the use of SACT for the treatment of advanced melanoma in Ireland [6]. Several of these are approved for reimbursement by the HSE, including pembrolizumab, ipilimumab, nivolumab, and multiple agents for BRAF V600 mutation-positive unresectable or metastatic melanoma [7]. We discuss our experience of metastatic melanoma and treatment at a university hospital in the Mid-West of Ireland.

\section{Aims}

The aim of our study was to assess the incidence and clinicopathological features of metastatic melanoma in our centre, and subsequent treatment with SACT over a 6-year period.

\section{Methods}

We completed a retrospective review of all cases of melanoma diagnosed in our centre over a 6-year period from January 2014 to December 2019. A secure, computer-generated database, maintained by the pathology department in conjunction with melanoma multidisciplinary meetings, was the source of data for the purposes of this study. This data included demographics and clinical details of all patients diagnosed with melanoma in our centre during this period. All cases of stage IV metastatic disease were included in the study, as determined by presence of distant metastasis to skin, soft tissue or non-regional lymph node and/or distant metastasis to lung, brain, liver or other visceral sites. Additional clinical information was obtained from chart review. Histopathological details from primary lesion excision including site, Breslow thickness and pathological stage were assessed. Clinical stage at the time of initial investigations was recorded, determined based on wide local excision, sentinel lymph node biopsy, and/or radiological findings where applicable. Subsequent updates relating to disease progression detected at clinical follow up, or on subsequent radiological investigations, and treatment with oncology services were analysed.

\section{Results}

\section{Demographics and clinical characteristics}

Six hundred twenty patients with melanoma were identified during this period from January 2014 to December 2019.
Twenty-eight (4.5\%) were diagnosed with metastatic melanoma during the study period, with 20 of these (71\%) being male patients. Mean age at primary diagnosis was 64.5 years (range 24-90 years).

Median Breslow depth at presentation was $4.3 \mathrm{~mm}$. Mean Breslow depth was $5.5 \mathrm{~mm}, \mathrm{SD} \pm 4.4 \mathrm{~mm}$. Further details of demographics, site of primary melanoma and histological characteristics are outlined in Table 1.

Seventy-one percent $(n=20)$ were tested for BRAF mutations, of which $20 \%(n=4)$ were positive.

\section{Clinical stage after initial diagnosis}

Thirteen patients (46\%) had evidence of metastases at the time of initial workup. Nine patients $(69 \%)$ had clinically detected distant cutaneous metastases at presentation. Five (38\%) had distant metastases detected on radiological staging. The clinical stage at time of initial investigations and time to detection of metastases is outlined in Table 2, based on the American Joint Committee on Cancer (AJCC) staging manual 8th edition post-2018 and 7th edition pre-2018 $[8,9]$. For the other 15 patients with earlier stage melanoma at presentation, mean time to detection of metastases was 2.9 years.

\section{Systemic anti-cancer treatment}

Fifteen patients (53\%) received SACT. Ten patients received immunotherapy with checkpoint inhibitors, 3 received combined BRAF and MEK inhibitor therapy, and 1 had BRAF inhibitor monotherapy. One patient received interferon. Of the 13 patients who did not receive systemic treatment, 8 had radiological and clinical surveillance alone, and 3 declined

Table 1 Demographics and clinical characteristics

\begin{tabular}{ll}
\hline Total patients with metastasis & 28 \\
Sex, male (\%) & $20(71)$ \\
Age, years (range) & $64.7(24-90)$ \\
Site of primary melanoma & Number (\%) \\
Head and neck & $10(34)$ \\
Upper limb & $4(14)$ \\
Lower limb, number & $7(28)$ \\
Torso, number & $3(10)$ \\
Unknown primary melanoma & $4(14)$ \\
Primary melanoma histological characteristics & \\
Mean Breslow depth, mm (range) & \\
Median Breslow depth, mm & $5.5(0-20)$ \\
Ulceration present (\%) & 4.3 \\
Mitotic rate, per mm ${ }^{2}$ (range) & $7(25)$ \\
Regression identified (\%) & $3.6(0-18)$ \\
\hline
\end{tabular}

${ }^{\mathrm{a}}$ Where available, excluding unknown primary lesions 
Table 2 AJCC clinical stage at initial diagnosis of patients who developed metastatic disease

\begin{tabular}{lll}
\hline Stage & $\begin{array}{l}\text { Number of } \\
\text { patients }(\%)\end{array}$ & $\begin{array}{l}\text { Mean time to detection of metasta- } \\
\text { sis after initial investigations, years }\end{array}$ \\
\hline IV & $13(46 \%)$ & - \\
III (A, B, C) & $5(17 \%)$ & 1.9 \\
II (A, B, C) & $7(24 \%)$ & 2.3 \\
I & $3(10 \%)$ & 6.2 \\
\hline
\end{tabular}

further treatment or surveillance. Two patients got referred to other hospitals and were followed up there. Five patients had interval radiological improvement of metastatic disease after commencing SACT, which remained stable on all follow-up imaging. At the time of data analysis, these had remained stable, with an average period of 2.2 years of follow-up (range 0.9-4.4 years). One patient had cutaneous metastases which resolved on commencing SACT and remained free of metastases on all subsequent assessments over a 4-year period.

Eleven of 28 patients died, with mean age at death of 80.2 years (range $71-94$ ). Median overall survival from the diagnosis of melanoma was 1.5 years $(S D \pm 1.3$ years). Five of these patients had received SACT. Medial overall survival from diagnosis for patients who had SACT was 2.8 years $(\mathrm{SD} \pm 1.1)$ compared to 1.2 years $(\mathrm{SD} \pm 0.9)$ for those without systemic treatment. A comparison of these two groups is outlined in Table 3.

\section{Discussion}

Almost half of patients with metastatic melanoma had advanced disease at the time of initial diagnosis in our centre. This may be due to a significant delay in diagnosis or represent aggressive disease biology. The male predominance in this patient group is reflected in national data, with a higher proportion of advanced melanomas seen in males [1]. BRAF mutation testing was performed for the majority (71\%) of patients with metastatic melanoma. BRAF testing is typically carried out on the primary pathology sample, though other tissues such as from metastatic deposits may also be used. BRAF mutations are typically found in approximately $50 \%$ of advanced melanomas, with the $V 600 E$ mutation most commonly observed [10]. Though the sample size included in this study was small, the proportion of $B R A F$ mutation-positive melanomas was relatively low in comparison with international studies.

Treatment of advanced melanoma has undergone dramatic changes in the last decade, with predictable therapeutic efficacy in particular with targeted therapies for $B R A F$ positive melanomas [11]. While the majority of patients with metastatic melanoma in our centre received SACT, just under half did not. Factors precluding systemic treatment were related to comorbidities, poor performance status due to very advanced disease, or patient preference. These factors however were not consistently documented or formally studied as part of this analysis, and further research in this area may be beneficial in optimising treatment pathways for late-stage melanoma. Toxicity with treatment, for example, is a significant factor considered by oncologists when exploring options with patients with advanced melanoma.

For those with multiple comorbidities or poor baseline health, systemic therapies may reduce overall quality of life due to adverse effects of treatment. Thus, each treatment plan is individualised carefully. Recent publications highlight the need for further research to optimise treatment for advanced melanoma, with focus on predictive and diagnostic biomarkers, improving treatment tolerability and potential treatment duration [12].

The economic cost and availability of SACT are further factors that may limit management in advanced melanoma. These agents are expensive and their introduction presents a challenge considering national health budget constraints. The available treatments in addition can differ across the European Union, with some countries approving reimbursement for SACT sooner than others after licensing approval by the European Medicines Agency. Access to cancer treatments in the Irish public health service is generally equal to that within private health system; however, circumstances have been observed in which private health insurers made treatments available for a greater number of indications. For example, pembrolizumab, a monoclonal anti-programmed cell death-1 (PD-1) antibody, has been reimbursed by the HSE as monotherapy for the treatment of adults with unresectable or advanced melanoma since June 2016. The HSE commissioned pharmacoeconomic
Table 3 Survival time in patients who received SACT for metastatic melanoma compared with those who did not receive SACT

\begin{tabular}{llll}
\hline & $\begin{array}{l}\text { Patients that } \\
\text { received SACT }\end{array}$ & $\begin{array}{l}\text { Patients that did not } \\
\text { receive SACT }\end{array}$ & $\begin{array}{l}\text { Both } \\
\text { groups } \\
\text { combined }\end{array}$ \\
\hline $\begin{array}{l}\text { Number of patient deaths } \\
\begin{array}{l}\text { Median overall survival time from time } \\
\text { of diagnosis to death, years }\end{array}\end{array}$ & 5 & 6 & 11 \\
Median age at death, years & 78.7 & 1.2 & 1.5 \\
\hline
\end{tabular}


assessment of pembrolizumab for adjuvant melanoma treatment with the National Centre for Pharmacoeconomics (NCPE) in December 2018 [13]. In 2019, VHI, a private health insurance company, began funding several cancer treatments prior to their approval for public reimbursement. These included pembrolizumab for adjuvant treatment of stage III melanoma. For patients without VHI cover, the treatment remained available for stage IV disease only. The divide in availability of these cancer treatments was widely covered in national news media $[14,15]$. Funding was made available by the HSE for this indication in May 2021.

This study analysed data from 2014 to 2019 , prior to significant changes in health systems nationally in March 2020. Since the onset of the COVID-19 pandemic, a pattern of fewer referrals, patient hesitancy to seek medical care, along with exacerbated clinical delays has been observed [16]. This is a concerning trend in the context of diagnostic delay of melanoma. In our centre, an average of 115 new patients with melanoma had been diagnosed per year up until 2019; however, this dropped over twofold to just 53 in 2020. As the pandemic emergency measures ease, priority is moving towards reduction of waiting lists particularly for urgent referrals. Additional clinics have been established, with a higher proportion of these dedicated to pigmented lesion assessment. In our centre, the introduction of these clinics in 2012, aimed at early detection of melanoma, has been associated with an increase in the number of melanomas excised [17].

In summary, melanoma is increasing in incidence and presentation with advanced disease is not uncommon. As incidence increases, so too will the number of patients who develop metastatic disease. While options for systemic treatment are continuing to expand and offer improved outcomes, further research is needed on factors preventing SACT use and improving toxicity and duration of these therapies. Primary prevention and early diagnosis of melanoma remain key areas of focus. Disease awareness is essential amongst both medical professional and the general population in the timely detection of melanoma, and particularly the reduction of metastatic disease.

Author contribution All authors contributed to the study conception and design. Material preparation and data collection were performed by EP and VM. EP analysed the data and prepared the manuscript. All authors read and approved the final manuscript.
Code availability Not applicable.

\section{Declarations}

Ethics approval This was an observational study conducted retrospectively from data obtained for clinical purposes as part of departmental audit. The University of Limerick Hospital Group Research Ethics Committee has confirmed that no ethical approval is required.

Consent to participate Not applicable.

Consent for publication Not applicable.

Conflict of interest The authors declare no competing interests.

\section{References}

1. National Cancer Registry Ireland (2017) Cancer trends no. 34 - skin cancer. https://www.ncri.ie/sites/ncri/files/pubs/Trends\% 20report\%20skin\%20cancer\%20final180717.pdf. Accessed 1 August 2021

2. European Cancer Observatory (ECO), EUCAN database. Rates standardised to the European (1976) standard population. http:// eco.iarc.fr/EUCAN/Cancer.aspx?Cancer=20. Accessed 1 August 2021

3. Kudchadkar RR et al (2020) Metastatic melanoma. CA A Cancer J Clin 70:78-85

4. Luke JJ et al (2017) Targeted agents and immunotherapies: optimizing outcomes in melanoma. Nat Rev Clin Oncol 14(8):463-482

5. Michielin O et al (2020) Evolving impact of long-term survival results on metastatic melanoma treatment. Journal for ImmunoTherapy of Cancer 8:e000948

6. National Cancer Control Programme (2017) Systemic anti-cancer therapy of patients with metastatic melanoma: Evidence into Practice. https://www.hse.ie/eng/services/list/5/cancer/profinfo/guidelines/ eiprr\%20melanoma.pdf. Accessed 1 August 2021

7. Health Service Executive. Cancer Drugs Approved for Reimbursement. Available at: https://www.hse.ie/eng/services/list/5/cancer/ profinfo/medonc/cdmp/new.html. Accessed 1 August 2021

8. Keung EZ, Gershenwald JE (2018) The eighth edition American Joint Committee on Cancer (AJCC) melanoma staging system: implications for melanoma treatment and care. Expert Rev Anticancer Ther 18(8):775-784

9. Edge SB, Compton CC (2010) The American Joint Committee on Cancer: the 7th edition of the AJCC cancer staging manual and the future of TNM. Ann Surg Oncol 17(6):1471-1474

10. Cheng L et al (2018) Molecular testing for BRAF mutations to inform melanoma treatment decisions: a move toward precision medicine. Mod Pathol 31(1):24-38

11. Jenkins RW, Fisher DE (2021) Treatment of Advanced Melanoma in 2020 and Beyond. J Invest Dermatol 141(1):23-31

12. Weiss SA, Wolchok JD, Sznol M (2019) Immunotherapy of melanoma: facts and hopes. Clin Cancer Res 25(17):5191-5201 
13. National Centre for Pharmacoeconomics. Pembrolizumab for adjuvant melanoma. www.ncpe.ie/drugs/pembrolizumab-keytruda-foradjuvant-melanoma/. Accessed 1 August 2021

14. Irish Times (2019) Rivals have no plans to follow VHI's decision on cancer drugs. www.irishtimes.com/news/health/rivals-haveno-plans-to-follow-vhi-s-decision-on-cancer-drugs-1.3861304. Accessed 1 August 2021

15. Irish Independent (2019) Three-tier divide opens up as VHI covers its private patients' costs for top cancer drugs. https:// www.independent.ie/irish-news/health/three-tier-divide-opensup-as-vhi-covers-its-private-patients-costs-for-top-cancerdrugs-38019573.html. Accessed 1 August 2021

16. Dessinioti C, Garbe C, Stratigos AJ (2021) The impact of the COVID-19 pandemic on diagnostic delay of skin cancer: a call to restart screening activities [published online ahead of print, 2021 Jul 26]. J Eur Acad Dermatol Venereol. https://doi.org/10.1111/ jdv. 17552

17. Lynch $\mathrm{M}$ et al (2017) Melanoma diagnosis and management after the introduction of a pigmented lesion clinic in the Mid-West of Ireland. Ir J Med Sci 186:671-675

Publisher's Note Springer Nature remains neutral with regard to jurisdictional claims in published maps and institutional affiliations. 